\title{
Melting behavior of alkaline-earth metal carbodiimides and their thermochemistry from first principles
}

Akira Hosono ${ }^{1, *}$, Ralf Peter Stoffel ${ }^{2}$, Yuji Masubuchi ${ }^{3, *}$, Richard Dronskowski²,4, Shinichi Kikkawa ${ }^{3}$

Affiliations;

${ }^{1}$ Graduate School of Chemical Science and Engineering, Hokkaido University, N13W8, Kita-ku, Sapporo 060-8628, Japan

${ }^{2}$ Chair of Solid-State and Quantum Chemistry, Institute of Inorganic Chemistry, RWTH Aachen University, 52056 Aachen, Germany

${ }^{3}$ Faculty of Engineering, Hokkaido University, N13W8, Kita-ku, Sapporo 060-8628, Japan

${ }^{4}$ Hoffmann Institute of Advanced Materials, Shenzhen Polytechnic, 7098 Liuxian Boulevard, Nanshan District, Shenzhen, China

*Corresponding authors:

Akira Hosono; address: Graduate School of Chemical Science and Engineering, Hokkaido University, N13W8, Kita-ku, Sapporo 060-8628, Japan; Tel/Fax: +81-(0)11706-6739/6740; E-mail: ezohakitaguni@frontier.hokudai.ac.jp

Associate professor Yuji Masubuchi; address: Faculty of Engineering, Hokkaido University, N13W8, Kita-ku, Sapporo 060-8628, Japan; Tel: +81-(0)11-706-6742; Email: yuji-mas@eng.hokudai.ac.jp

\section{Content}

Figure S1 Thermogravimetry curve of CaNCN powder. 


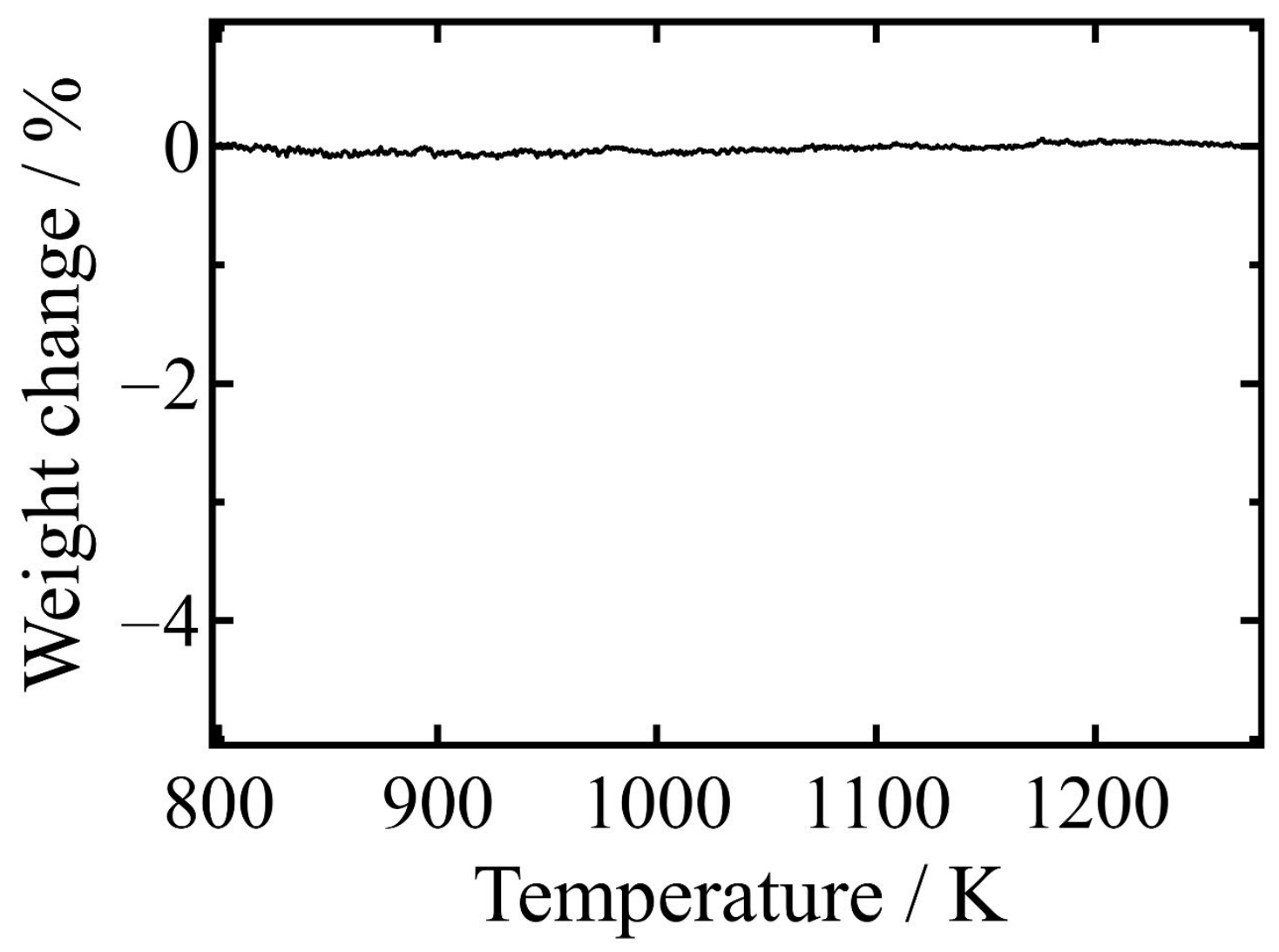

Figure S1 Thermogravimetry curve of CaNCN powder contaminated by approximately $18 \mathrm{wt} \%$ of $\mathrm{CaO}$. The sample was heated at a rate of $10 \mathrm{~K} / \mathrm{min}$ under flowing nitrogen. 